\title{
CDISC SDTM Priority of Tuberculosis Contact Terminology
}

National Cancer Institute

\section{Source}

National Cancer Institute. CDISC SDTM Priority of Tuberculosis Contact Terminology. NCI Thesaurus. Code C102582.

Terminology associated with the priority of tuberculosis contact codelist of the Clinical Data Interchange Standards Consortium (CDISC) Study Data Tabulation Model (SDT M). 